\title{
Valuation theory of exponential Hardy fields I*
}

\author{
Franz-Viktor and Salma Kuhlmann \\ Dedicated to Murray Marshall on the occasion of his 60th birthday
}

11. 4.2002

\section{Introduction}

In this paper, we analyze the structure of the Hardy fields associated with o-minimal expansions of the reals with exponential function. In fact, we work in the following more general setting. We take $T$ to be the theory of a polynomially bounded o-minimal expansion $\mathcal{P}$ of the ordered field of real numbers. By $\mathcal{F}_{T}$ we denote the set of all 0-definable functions of $\mathcal{P}$. Further, we assume that $T$ defines the restricted exponential and logarithmic functions (cf. [D-M-M1]). Then also $T(\exp )$ is o-minimal (cf. $[\mathrm{D}-\mathrm{S} 2])$. Here, $T(\exp )$ denotes the theory of the expansion $(\mathcal{P}$, exp) where exp is the un-restricted real exponential function. Finally, we take any model $\mathcal{R}$ of $T(\exp )$ which contains $\left(\mathbb{R},+, \cdot,<, \mathcal{F}_{T}\right.$, exp) as a substructure. Then we consider the Hardy field $H(\mathcal{R})$ (see Section 2.2 for the definition) as a field equipped with convex valuations. Theorem $\mathrm{B}$ of [D-S2] tells us that $T(\exp )$ admits quantifier elimination and a universal axiomatization in the language augmented by a symbol for the inverse function log of exp. This implies that $H(\mathcal{R})$ is equal to the closure of its subfield $\mathcal{R}(x)$ under $\mathcal{F}_{T}$, exp and $\log$; here, $x$ denotes the germ of the identity function (cf. [D-M-M1], §5; the arguments also hold in the case where $\mathcal{R}$ is a non-archimedean model).

We shall analyze the valuation theoretical structure of this closure by explicitly showing how it can be built up from $\mathcal{R}(x)$ (cf. Section 3.3). Our construction method yields the following result (see Section 3.4 for definitions):

Theorem 1.1 Every model $\mathcal{R}$ as chosen above is levelled.

This implies that $T(\exp )$ has levels with parameters, in the sense of $[\mathrm{M}-\mathrm{M}]$, and is exponentially bounded (cf. Theorem 3.11). We can determine the level of a function explicitly: it is the difference of two numbers which come up naturally in our construction method.

In Section 3.5 we use our main structure theorem (Theorem 3.11) to deduce:

*This paper was written while both authors were partially supported by a Canadian NSERC research grant. 
Theorem 1.2 Suppose that for all $r \in \mathbb{R}, \mathcal{F}_{T}$ contains the power function

$$
\begin{aligned}
P_{r}:(0, \infty) & \longrightarrow \mathbb{R} \\
x & \mapsto x^{r} .
\end{aligned}
$$

Let $\mathcal{R}_{T}$ denote the reduct of $\mathcal{R}$ to the language of $T$. Then the Hardy field $H\left(\mathcal{R}_{T}\right)$ is maximal among the Hardy subfields of $H(\mathcal{R})$ associated with polynomially bounded reducts of $\mathcal{R}$.

L. v. d. Dries conjectured that

$$
\mathbb{R}_{\text {an,powers }}=\left(\mathbb{R},+, \cdot, 0,1,<, \mathcal{F}_{\text {an }},\left\{P_{r} \mid r \in \mathbb{R}\right\}\right),
$$

the expansion of the ordered field of real numbers by the set $\mathcal{F}_{\text {an }}$ of restricted analytic functions and the power functions $P_{r}$, is a maximal polynomially bounded reduct of

$$
\mathbb{R}_{\text {an }, \exp }=\left(\mathbb{R},+, \cdot, 0,1,<, \mathcal{F}_{\text {an }}, \exp \right),
$$

At least on the level of Hardy fields, this is true: since the elementary theory of $\mathbb{R}_{\text {an,powers }}$ is polynomially bounded and o-minimal and the power functions are definable in $\mathbb{R}_{\text {an,exp }}$ (cf. $[\mathrm{M}]$ ), the foregoing theorem shows (cf. Theorem 3.16 for a more general result): $H\left(\mathbb{R}_{\text {an,powers }}\right)$ is maximal among the Hardy subfields of $H\left(\mathbb{R}_{\mathrm{an}, \exp }\right)$ associated with polynomially bounded reducts of $\mathbb{R}_{\mathrm{an}, \exp }$.

In a subsequent paper, we shall study the residue fields of exponential Hardy fields with respect to arbitrary convex valuations (which are not necessarily $T$ (exp)-convex).

\section{Some preliminaries}

If $(K, w)$ is a valued field, then we write $w a$ for the value of $a \in K$ and $w K$ for its value group $\{w a \mid 0 \neq a \in K\}$. Further, we write $a w$ for the residue of $a$, and $K w$ for the residue field. The valuation ring is denoted by $\mathcal{O}_{w}$. For generalities on valuation theory, see $[R]$. For the general notions and tools from model theory we use in this paper, we refer the reader to $[\mathrm{C}-\mathrm{K}]$.

\subsection{Convex valuations}

A valuation $w$ on an ordered field $K$ is called convex if $\mathcal{O}_{w}$ is convex. The convex valuation rings of an ordered field are linearly ordered by inclusion. If $\mathcal{O}_{w} \varsubsetneqq \mathcal{O}_{w^{\prime}}$ then $w$ is said to be finer than $w^{\prime}$. There is always a finest convex valuation, called the natural valuation. It is characterized by the fact that its residue field is archimedean. A valuation $w$ on an ordered field is convex if and only if the natural valuation is finer or equal to $w$. Throughout this paper, $v$ will always denote the natural valuation, unless stated otherwise. 
If $a, b$ are elements of an ordered group or an ordered field, then we write $a \ll b<0$ if $a<b<0$ and $\forall n \in \mathbb{N}: a<n b$. Similarly, $a \gg b>0$ if $a>b>0$ and $\forall n \in \mathbb{N}: a>n b$. We set $|a|:=\max \{a,-a\}$. Then the natural valuation is characterized by:

$$
v a<v b \Leftrightarrow|a| \gg|b| .
$$

Note that if $\mathbb{R} \subset K$ and $a \in K$ with $v a=0$, then there is some $r \in \mathbb{R}$ such that $v(a-r)>0$. Further, $w r=0$ for every $r \in \mathbb{R}$ and every convex valuation $w$.

Lemma 2.1 Let $v, w$ be arbitrary valuations on some field $K$. Suppose that $v$ is finer than $w$. Then for all $a, b \in K$,

$$
v a \leq v b \Rightarrow w a \leq w b .
$$

In particular, $w a>0 \Rightarrow v a>0$. Further, $H_{w}:=\{v z \mid z \in K \wedge w z=0\}$ is a convex subgroup of the value group $v K$ of $v$. We have that $v z \in H_{w} \Leftrightarrow z \in \mathcal{O}_{w}^{\times}$. There is a canonical isomorphism $w K \simeq v K / H_{w}$. Conversely, every convex subgroup of $v K$ is of the form $H_{w}$ for some valuation $w$ such that $v$ finer or equal to $w$.

The valuation $v$ of $K$ induces a valuation $v / w$ on $K w$. There are canonical isomorphisms $v / w(K w) \simeq H_{w}$ and $(K w) v / w \simeq K v$. If $K w$ is embedded in $\mathcal{O}_{w}$ such that the restriction of the residue map is the identity on $K w$, then $v / w=\left.v\right|_{K w}$ (up to equivalence). Writing $v$ instead of $\left.v\right|_{K w}$, we then have that $v(K w)=H_{w}$ and $(K w) v=K v$.

We will call $H_{w}$ the convex subgroup associated with $w$ and $w$ the valuation associated with $H_{w}$. Since the isomorphism is canonical, we will write $w K=v K / H_{w}$.

The order type of the chain of nontrivial convex subgroups of an ordered abelian group $G$ is called the rank of $G$. If finite, then the rank is not bigger than the maximal number of rationally independent elements in $G$. In particular, $G$ has finite rank if it is finitely generated or equivalently, if its divisible hull is a $\mathbb{Q}$-vector space of finite dimension.

From (1) and (2) it follows that for every convex valuation $w$,

$$
|a| \leq|b| \Rightarrow w a \geq w b .
$$

For the rest of this section, we will assume that $(M, \exp )$ is a model of the elementary theory of $(\mathbb{R},+, \cdot, 0,1,<, \exp )$ such that $\mathbb{R} \subset M$ and the restriction of $\exp$ to $\mathbb{R}$ is the natural exponential exp on $\mathbb{R}$. Further, we take $w$ to be any convex valuation on $M$. Then the exponential exp of $M$ is an order preserving isomorphism from the additive group of $M$ onto its multiplicative group of positive elements. Its inverse is the logarithm log; it is order preserving and defined for all positive elements. Consequently, if $z \in M$ is positive infinite, that is, $z>\mathbb{R}$, then $\log z>\log (\{r \in \mathbb{R} \mid r>0\})=\mathbb{R}$. In other words,

$$
v z<0 \wedge z>0 \Rightarrow v \log z<0 \wedge \log z>0 .
$$

Further, exp satisfies the Taylor axiom scheme:

$$
|z| \leq 1 \Rightarrow\left|\exp z-\sum_{n=0}^{m} \frac{z^{n}}{n !}\right|<\left|z^{m}\right| \quad(m \in \mathbb{N}) .
$$


In order to derive a valuation theoretical property from this axiom, we need the following simple lemma:

Lemma 2.2 Let $K$ be an ordered field and $w$ a convex valuation on $K$. Suppose that $h \in K$ satisfies

$$
\left|h-\sum_{k=0}^{m} s_{k} z_{k}\right|<\left|s_{m}^{\prime} z_{m}\right| \quad \text { for all } m \in \mathbb{N},
$$

where $s_{k}, s_{k}^{\prime} \in \mathbb{R} \backslash\{0\}$, and $z_{k} \in K$ are such that $w z_{k+1}>w z_{k}$. Write

$$
S_{m}:=\sum_{k=0}^{m} s_{k} z_{k}
$$

Then $\left(S_{m}\right)_{m \in \mathbb{N}}$ is a pseudo Cauchy sequence in $(K, w)$. Further,

$$
w\left(h-S_{m}\right)=w z_{m+1}=w\left(S_{m+1}-S_{m}\right),
$$

which shows that $h$ is a limit of this sequence.

Proof: $\quad$ Recall that $w s=0$ for $0 \neq s \in \mathbb{R}$, and that $w|a|=w a$ for every $a$ in $K$. By (5) and (3), we have that

$$
\begin{aligned}
w\left(h-S_{m}-s_{m+1} z_{m+1}-s_{m+2} z_{m+2}\right) & =w\left(h-S_{m+2}\right) \geq w s_{m+2}^{\prime} z_{m+2}=w z_{m+2} \\
& >w z_{m+1}=w s_{m+1} z_{m+1} .
\end{aligned}
$$

By the ultrametric triangle law,

$$
w\left(s_{m+1} z_{m+1}+s_{m+2} z_{m+2}\right)=\min \left\{w s_{m+1} z_{m+1}, w s_{m+2} z_{m+2}\right\}=w s_{m+1} z_{m+1} .
$$

Hence, again by the ultrametric triangle law,

$$
\begin{aligned}
w\left(h-S_{m}\right) & =\min \left\{w\left(h-S_{m}-s_{m+1} z_{m+1}-s_{m+2} z_{m+2}\right), w\left(s_{m+1} z_{m+1}+s_{m+2} z_{m+2}\right)\right\} \\
& =w s_{m+1} z_{m+1}=w\left(S_{m+1}-S_{m}\right) .
\end{aligned}
$$

Lemma 2.3 For every $z \in M$,

$$
\begin{aligned}
& w z>0 \Rightarrow w \exp z=0 \wedge w(\exp z-1)=w z \\
& v z=0 \Rightarrow v \exp z=0 .
\end{aligned}
$$

Proof: By Lemma 2.1, wz>0 implies $v z>0$, that is, $z$ is infinitesimal. In particular, $|z|<1$, and (TA) holds. Applying (6) of Lemma 2.2 with $m=1$ and $z_{m}=z^{m}$, we find that $w(\exp z-1-z)=w z^{2}=2 w z>w z$. By the ultrametric triangle law, this implies that $w \exp z=w(1+z)=w 1=0$ and $w(\exp z-1)=w z$. This proves $(7)$.

Now assume that $v z=0$. Then there is some $r \in \mathbb{R} \subset M$ such that $v(z-r)>0$. We have that $\exp r \in \mathbb{R}$, hence $v \exp r=0$. By (7) with $w=v, v \exp (z-r)=0$. Thus, $v \exp z=v \exp r \exp (z-r)=v \exp r+v \exp (z-r)=0$. This proves (8).

With $M$ as before, exp also satisfies the following growth axiom scheme: 


$$
z>m^{2} \Longrightarrow \exp z>z^{m} \quad(m \in \mathbb{N})
$$

From this, we derive:

Lemma 2.4 For every $z \in M$,

$$
\begin{aligned}
w z<0 \wedge z>0 & \Rightarrow w \exp z \ll w z \ll w \log z<0 \\
w z=0 \wedge z>0 & \Rightarrow w \log z \geq 0 \\
v z \geq 0 & \Leftrightarrow v \exp z=0 .
\end{aligned}
$$

Proof: $\quad$ If $w z<0$ and $z>0$, then $z>\mathbb{R}$ and thus, $z>m^{2}$ for every $m \in \mathbb{N}$. So by (GA), $\exp z>z^{m}>0$ for all $m$. Hence by (3), $w \exp z \leq m w z$ for all $m$, i.e., $w \exp z \ll w z<0$. In view of (4), we can replace $z$ by $\log z$ to get that $w z \ll w \log z<0$. This proves (9).

Now assume that $w z=0$ and $z>0$. If $v z<0$, then by (9), $v z<v \log z<0$. If $v z>0$, then $v z^{-1}<0$ and by $(9), v z^{-1}<v \log z^{-1}=v(-\log z)=v \log z<0$. In both cases, it follows from Lemma 2.1 that $0=w z=w z^{-1} \leq w \log z \leq 0$, i.e., $w \log z=0$. Now let $v z=0$. If $v \log z<0$, then by (9), $v z=v \exp \log z<0$ if $\log z>0$, and $v z=-v z^{-1}=-v \exp (-\log z)>0$ if $\log z<0$. Hence, $v \log z \geq 0$, and again by Lemma 2.1, $w \log z \geq 0$. This proves $(10)$.

Implication " $\Rightarrow$ " of (11) follows from (7) with $w=v$, together with (8). The converse implication follows from (10), where we take $w=v$ and replace $z$ by $\exp z$.

For positive infinite elements $z \in M$ and $m \in \mathbb{Z}$, we set $\log _{0} z=z, \log _{m+1} z=$ $\log \left(\log _{m} z\right)$ if $m \geq 0$, and and $\log _{m-1} z=\exp \left(\log _{m} z\right)$ if $m \leq 0$; note that every $\log _{m} z$ is again positive infinite. Similarly, we define $\exp _{m} z$ for every $z \in M$.

Corollary 2.5 Assume that $\mathcal{R}$ is an exp-closed subfield of $M$. If $x \in M$ such that $w x<w \mathcal{R}$ and $x>0$, then for $m>1$,

$$
w x \ll w \log x \ll \ldots \ll w \log _{m} x \ll \ldots<w \mathcal{R} .
$$

Proof: The part " $w x \ll w \log x \ll \ldots \ll w \log _{m} x$ " follows from (9) by induction on $m$. Now suppose that there is a positive integer $m$ and some $\alpha \in w \mathcal{R}$ such that $\alpha \leq w \log _{m} x$. Replacing $\alpha$ by $2 \alpha \in w \mathcal{R}$ if necessary, we may assume that $\alpha<w \log _{m} x$. Take a positive element $a \in \mathcal{R}$ such that $w a=\alpha$. Then by virtue of $(3), 0<\log _{m} x<a$. It follows that $x<\exp _{m} a$, which implies that $w x \geq w \exp _{m} a \in w \mathcal{R}$. This proves that if $w x<w \mathcal{R}$ then $w \log _{m} x<w \mathcal{R}$ for all $m$.

For further details on the valuation theory of exponential fields, see [KS2], [KS1] and [K-K1]. 


\subsection{Hardy fields}

Let us recall some basic facts about Hardy fields. Initially, they were only defined as fields consisting of germs at $\infty$ of real-valued functions. But we will work with a more general definition that has also been used by other authors lately. Assume that $T$ is the theory of any o-minimal expansion of the ordered field of real numbers by real-valued functions, and that $\mathcal{R}$ is a model of $T$. The Hardy field of $\mathcal{R}$, denoted by $H(\mathcal{R})$, is the set of germs at $\infty$ of unary $\mathcal{R}$-definable functions $f: \mathcal{R} \rightarrow \mathcal{R}$. Then $H(\mathcal{R})$ is an ordered differential field which contains $\mathcal{R}$. Let $x \in H(\mathcal{R})$ be the germ of the identity function. Then $H(\mathcal{R})$ is the closure of $\mathcal{R}(x)$ under all 0 -definable functions of $\mathcal{R}$.

By $v_{\mathcal{R}}$ we will denote the finest convex valuation on $H(\mathcal{R})$ which is trivial on $\mathcal{R}$. Then $v_{\mathcal{R}} a<0$ if and only if $a>\mathcal{R}$. If $f, g$ are non-zero unary $\mathcal{R}$-definable functions on $\mathcal{R}$, then we will denote their germs in $H(\mathcal{R})$ by the same letters. With this convention, the following holds:

$$
v_{\mathcal{R}} f=v_{\mathcal{R}} g \Longleftrightarrow \lim _{x \rightarrow \infty} \frac{f(x)}{g(x)} \text { is a non-zero constant in } \mathcal{R} .
$$

(Note that " $x \rightarrow \infty$ " means letting $x$ outgrows every element of $\mathcal{R}$.) The functions $f$ and $g$ are asymptotic on $\mathcal{R}$ if and only if this constant is 1 , and we have:

$$
v_{\mathcal{R}}(f-g)>v_{\mathcal{R}} g \Longleftrightarrow f \text { and } g \text { are asymptotic on } \mathcal{R}
$$

or in other words,

$$
v\left(\frac{f}{g}-1\right)>v \mathcal{R} \Longleftrightarrow f \text { and } g \text { are asymptotic on } \mathcal{R},
$$

\section{Closures of $\mathcal{R}(x)$ under $\mathcal{F}, \log$ and exp}

General assumptions: Throughout this section, we will assume that $T$ is the theory of a polynomially bounded o-minimal expansion $\mathcal{P}$ of the ordered field of real numbers by real-valued functions. Further, we assume that $T$ defines the restricted exp and log. Then also $T(\exp )$ is o-minimal (cf. [D-S2]). Here, $T(\exp )$ denotes the theory of the expansion $(\mathcal{P}, \exp )$ where exp is the un-restricted real exponential function.

The archimedean field

$$
\mathrm{Q}:=\left\{r \in \mathbb{R} \mid \text { the function } x \mapsto x^{r}:(0, \infty) \longrightarrow \mathbb{R} \text { is } 0 \text {-definable in } \mathcal{P}\right\}
$$

is called the field of exponents of $T$.

We let $\mathcal{F}_{T}$ denote the set of function symbols in the language of $T$ and assume that there is a function symbol in $\mathcal{F}_{T}$ for each 0 -definable function of $\mathcal{P}$. This implies that $T$ admits quantifier elimination and a universal axiomatization (cf. [D-L], §2). We let $\mathcal{F}$ denote any subset of $\mathcal{F}_{T}$.

Further, we assume that $M$ is a model of $T$. (Later, we will assume that it is a model of $T(\exp )$, but we will not distinguish between this model and its reduct to the language 
of T.) Suppose that $K$ is a submodel (hence an elementary substructure) of $M$. Take $x_{i} \in M, i \in I$. By $K\left\langle x_{i} \mid i \in I\right\rangle$ we denote the 0-definable closure of $K \cup\left\{x_{i} \mid i \in I\right\}$ in $M$. By our assumption on the language of $T$, this is the closure of $K \cup\left\{x_{i} \mid i \in I\right\}$ under $\mathcal{F}_{T}$, that is, the smallest subfield of $M$ containing $K \cup\left\{x_{i} \mid i \in I\right\}$ and closed under all functions which interpret the function symbols of $\mathcal{F}_{T}$ in $M$. Since $T$ admits a universal axiomatization and $K\left\langle x_{i} \mid i \in I\right\rangle$ is a substructure of $M$, it is a model of $T$. Since $T$ admits quantifier elimination, $K\left\langle x_{i} \mid i \in I\right\rangle$ is an elementary substructure of $M$.

For an arbitrary subfield $F \subset M$, we let $F^{\mathrm{Q}}$ denote the smallest subfield of $M$ which contains $F$ and is Q-closed, i.e., closed under the exponents from Q. Further, we let $F^{\mathrm{rQ} \mathcal{F}}$ denote the smallest real closed subfield of $M$ which contains $F$, is Q-closed, and is $\mathcal{F}$-closed, i.e., closed under all functions on $M$ which are interpretations of function symbols in $\mathcal{F}$. We will say that $F$ is $\mathrm{rQ} \mathcal{F}$-closed if $F=F^{\mathrm{rQF}}$. Note that real closures can be taken to lie in $M$ since $M$ is real closed.

If $F$ is $\mathrm{Q}$-closed, then for every convex valuation $w$, the value group $w F$ is a Q-vector space with scalar multiplication defined by $q w(a)=w\left(|a|^{q}\right)$ for $q \in \mathrm{Q}$. If $\alpha \in w F$, then $\mathrm{Q} \alpha$ shall denote the $\mathrm{Q}$-subvector space generated by $\alpha$. As $\mathrm{Q}$ always contains $\mathbb{Q}$, we see that $w F^{\mathrm{Q}}$ is always divisible.

\subsection{Value groups}

The following property (Lemma 3.1) of polynomially bounded o-minimal expansions of the reals was proved in full generality in [D] (Lemma 5.4); see also Corollary 3.7 of [D-MM1]. Note that in the case of a polynomially bounded expansion, every convex valuation $w$ of a model is T-convex (cf. [D-L], §4).

Lemma 3.1 Assume that $\mathcal{R}$ is a submodel of $M$. If $x \in M$ such that $w x \notin w \mathcal{R}$, then $w \mathcal{R}\langle x\rangle=w \mathcal{R} \oplus \mathrm{Q} w x$.

Lemma 3.2 Assume that $\mathcal{R}$ is a submodel of $M$. Take elements $x_{i} \in M, i \in I$, such that the values $w x_{i}, i \in I$, are Q-linearly independent over $w \mathcal{R}$. Then

$$
w \mathcal{R}\left(x_{i} \mid i \in I\right)^{\mathrm{rQ \mathcal {F }}}=w \mathcal{R}\left(x_{i} \mid i \in I\right)^{\mathrm{Q}}=w \mathcal{R} \oplus \bigoplus_{i \in I} \mathrm{Q} w x_{i}
$$

Proof: $\quad$ Since every element of $\mathcal{R}\left(x_{i} \mid i \in I\right)^{\mathrm{rQ \mathcal {F }}}$ already lies in $\mathcal{R}\left(x_{i} \mid i \in I_{0}\right)^{\mathrm{rQ \mathcal {F }}}$ for a finite subset $I_{0} \subseteq I$ and a similar assertion is true for the fields $\mathcal{R}\left(x_{i} \mid i \in I\right)^{\mathrm{Q}}$ and $\mathcal{R}\left\langle x_{i} \mid i \in I\right\rangle$, it suffices to prove our assertion for the case of $I$ finite. We may write $I=\{1, \ldots, n\}$. By induction on $n$, Lemma 3.1 shows that

$$
w \mathcal{R}\left\langle x_{1}, \ldots, x_{n}\right\rangle=w \mathcal{R} \oplus \bigoplus_{i=1}^{n} \mathrm{Q} w x_{i} .
$$

Since $\mathcal{R}\left\langle x_{1}, \ldots, x_{n}\right\rangle$ is $\mathrm{rQ} \mathcal{F}$-closed, we have that

$$
\mathcal{R}\left(x_{1}, \ldots, x_{n}\right)^{\mathrm{Q}} \subseteq \mathcal{R}\left(x_{1}, \ldots, x_{n}\right)^{\mathrm{rQ \mathcal {F }}} \subseteq \mathcal{R}\left\langle x_{1}, \ldots, x_{n}\right\rangle .
$$


As $w \mathcal{R}\left(x_{1}, \ldots, x_{n}\right)^{\mathrm{Q}}$ is a $\mathrm{Q}$-vector space and contains $w x_{1}, \ldots, w x_{n}$, we obtain that

$$
\begin{aligned}
w \mathcal{R} \oplus \bigoplus_{i=1}^{n} \mathrm{Q} w x_{i} & \subseteq w \mathcal{R}\left(x_{1}, \ldots, x_{n}\right)^{\mathrm{Q}} \subseteq w \mathcal{R}\left(x_{1}, \ldots, x_{n}\right)^{\mathrm{rQ \mathcal {F }}} \\
& \subseteq w \mathcal{R}\left\langle x_{1}, \ldots, x_{n}\right\rangle=w \mathcal{R} \oplus \bigoplus_{i=1}^{n} \mathrm{Q} w x_{i}
\end{aligned}
$$

which shows that equality must hold everywhere.

\subsection{Linear independence of generating values}

From now on, let $M$ always be a model of $T(\exp )$, and $\mathcal{R}$ a submodel of $M$ containing $\left(\mathbb{R},+, \cdot,<, \mathcal{F}_{T}\right.$, exp) as a substructure. We take $\mathcal{F}$ as before, but always assume in addition that $\mathcal{F}$ contains function symbols for the restricted $\exp$ and $\log$. Hence, if a subfield $F$ of $M$ is $\mathcal{F}$-closed, then $\exp \varepsilon \in F$ and $\log (1+\varepsilon) \in F$ for every infinitesimal $\varepsilon$ in $F$. Since $\mathbb{R} \subseteq \mathcal{R}$, we have that $\mathcal{R} v=\mathbb{R}$.

Note that in view of Theorem $\mathrm{B}$ of $[\mathrm{D}-\mathrm{S} 2], \mathcal{R}$ is an elementary substructure of $M$, and $\left(\mathbb{R},+, \cdot,<, \mathcal{F}_{T}, \exp \right)$ is an elementary substructure of both. However, we will not use this fact in our constructions.

For every subfield $K$ of $\mathcal{O}_{w}$, its multiplicative group $K^{\times}$is contained in the multiplicative group $\mathcal{O}_{w}^{\times}$of all units of $\mathcal{O}_{w}$. We will say that $K$ is relatively exp-closed in $\mathcal{O}_{w}^{\times}$if $a \in K$ and $\exp (a) \in \mathcal{O}_{w}^{\times}$implies that $\exp (a) \in K$. For example, $\mathbb{R}$ is relatively exp-closed in $\mathcal{O}_{w}^{\times}$for every convex valuation $w$ of $M$.

Lemma 3.3 Let $K$ be a $\log$ - and $\mathrm{rQF}$-closed subfield of $M$. Let $w$ be a convex valuation of $M$. Assume that the residue field $K w$ is a subfield of $\mathcal{O}_{w} \cap K$, relatively exp-closed in $\mathcal{O}_{w}^{\times}$. Take any $a \in K$ such that $\exp a \notin K$. Then $w \exp a$ is Q-linearly independent over $w K$.

Proof: Suppose that $w \exp a$ is not Q-linearly independent over $w K$. Since $K$ is Qclosed, $w K$ is a Q-vector space, and it follows that $w \exp a=w b \in w K$ for some positive $b \in K$. Then $w \frac{\exp a}{b}=0$ and by Lemma 2.4, $w(a-\log b)=w \log \left(\frac{\exp a}{b}\right) \geq 0$. Since $K$ is $\log$-closed, $\log b \in K$. Hence, there is $c \in K w$ such that $w(a-\log b-c)>0$. By Lemma 2.3, this shows that $w \frac{\exp a}{b \exp c}=w \exp (a-\log b-c)=0$. In particular, we find that $w \exp c=w \frac{\exp a}{b}=0$, that is, $\exp c \in \mathcal{O}_{w}^{\times}$. By assumption on $K w, \exp c \in K w \subset K$.

By Lemma 2.1, $w(a-\log b-c)>0$ yields that $v(a-\log b-c)>0$. Therefore, $\exp (a-\log b-c) \in K^{\mathcal{F}}=K$, showing that $\exp a=\exp (a-\log b-c) \cdot b \cdot \exp c \in K$. We conclude: if $\exp a \notin K$, then $w \exp a$ is Q-linearly independent over $w K$.

Lemma 3.4 Assume that $K=\mathcal{R}\left(x_{i} \mid i \in I\right)^{\mathrm{rQF}} \subset M$ such that

1) the values $v x_{i}, i \in I$, are Q-linearly independent over $v \mathcal{R}$,

2) $x_{i}>0$ and $\log x_{i} \in K$ for all $i \in I$.

Then $K$ is log-closed. 
Proof: $\quad$ Take a positive $b \in K$. By virtue of Lemma 3.2, there is a finite subset $I_{0} \subset I$ and $q_{i} \in \mathrm{Q}$ such that $v b=v r^{\prime}+\sum_{i \in I_{0}} q_{i} v x_{i}$ for some positive $r^{\prime} \in \mathcal{R}$. So we can write $b=r^{\prime} \prod_{i \in I_{0}} x_{i}^{q_{i}} \cdot r \cdot(1+\varepsilon)$ with positive $r \in \mathbb{R}$ and some $\varepsilon \in K$ such that $v \varepsilon>0$. We have that $\log (1+\varepsilon) \in K$ since $K$ is $\mathcal{F}$-closed. Moreover, $\log r^{\prime} \in \mathcal{R} \subset K$ and $\log r \in \mathbb{R} \subset K$. Therefore,

$$
\log b=\log r^{\prime}+\sum_{i \in I_{0}} q_{i} \log x_{i}+\log r+\log (1+\varepsilon) \in K
$$

Lemma 3.5 Assume that $K$ is of the form

$$
\left.\begin{array}{l}
\mathcal{R}\left(x_{i} \mid i \in I\right)^{\mathrm{rQ} \mathcal{F}} \text { log-closed, with } x_{i}>0 \text { and } \\
v x_{i}, i \in I, \text { Q-linearly independent over } v \mathcal{R} .
\end{array}\right\}
$$

Take any $a \in K$ such that $\exp a \notin K$. Then $v \exp a$ is Q-linearly independent over $v K$,

$$
v K(\exp a)^{\mathrm{rQ \mathcal {F }}}=v K \oplus \mathrm{Q} v \exp a .
$$

Moreover, $K(\exp a)^{\mathrm{rQ \mathcal {F }}}$ is again log-closed, and therefore of the form (18). It contains $\exp b$ whenever $b \in K(\exp a)^{\mathrm{rQF}}$ and $v \exp b$ is Q-linearly dependent over $v K(\exp a)^{\mathrm{rQF}}$.

Proof: Applying Lemma 3.3 with $w=v$ and $K w=\mathbb{R}$, we obtain that $v \exp a$ is Q-linearly independent over $v K$ and that $\exp b \in K(\exp a)^{\mathrm{rQ} \mathcal{F}}$ whenever $b \in K(\exp a)^{\mathrm{rQ \mathcal {F }}}$ and $v \exp b$ is Q-linearly dependent over $v K(\exp a)^{\mathrm{rQF}}$. Equation (19) follows by an application of Lemma 3.2 to $K$ and to $K(\exp a)^{\mathrm{rQF}}$. Finally, we infer from Lemma 3.4 that $K(\exp a)^{\mathrm{rQ \mathcal {F }}}$ is log-closed.

Lemma 3.6 Assume that $(\mathcal{R}, v) \subset(K, v)$ is any extension of valued fields and that $w$ is a valuation on $K$ such that $v$ is finer than $w$, and $K w=\mathcal{R}$. Take $x_{i} \in K$ such that the values $v x_{i}, i \in I$, are Q-linearly independent over $v \mathcal{R}$. Then the values $w x_{i}, i \in I$, are Q-linearly independent.

Proof: $\quad$ From $K w=\mathcal{R}$ it follows that $v$ is the composition of $w$ with the restriction of $v$ to $\mathcal{R}$. Thus, $v \mathcal{R}$ is a convex subgroup of $v K$ and there is a canonical isomorphism $w K \simeq v K / v \mathcal{R}$. Hence $\sum_{i \in I} q_{i} w x_{i}=0$ (where $q_{i} \in \mathrm{Q}$, almost all of them zero) implies $\sum_{i \in I} q_{i} v x_{i} \in v \mathcal{R}$. By assumption, this implies that $q_{i}=0$ for all $i \in I$.

\subsection{A basic construction}

First, we show how to construct log-closed fields $K$ as in (18). From now on, we always assume that $x \in M$ such that $x>\mathcal{R}$, that is, $v x<v \mathcal{R}$ and $x>0$. By $v_{\mathcal{R}}$ we will denote the finest convex valuation on $M$ which is trivial on $\mathcal{R}$. 
Lemma 3.7 The field

$$
\mathcal{R}\left(\log _{m} x \mid m \geq 0\right)^{\mathrm{rQ \mathcal {F }}}
$$

is log-closed. The convex hull of its value group in $v M$ is equal to the smallest convex subgroup containing $v x$ and $v \mathcal{R}$. If $w$ is a convex valuation on $M$, trivial on $\mathcal{R}$ and such that $w x=0$, then the field $\mathcal{R}\left(\log _{m} x \mid m \geq 0\right)^{\mathrm{r} \mathrm{Q} \mathcal{F}}$ lies in $\mathcal{O}_{w}$.

Proof: From Corollary 2.5 we know that

$$
v x \ll v \log x \ll \ldots \ll v \log _{m} x \ll \ldots<v \mathcal{R} .
$$

In particular, the values $v \log _{m} x$ lie in distinct archimedean classes. As $\mathrm{Q}$ is archimedean, it follows that the values $v \log _{m} x$ are Q-linearly independent over $v \mathcal{R}$. So it follows from Lemma 3.4 that $\mathcal{R}\left(\log _{m} x \mid m \geq 0\right)^{\mathrm{r} Q \mathcal{F}}$ is log-closed.

From Lemma 3.2 we infer that $v \mathcal{R}\left(\log _{m} x \mid m \geq 0\right)^{\mathrm{r} \mathrm{Q} \mathcal{F}}=v \mathcal{R} \oplus \oplus_{m \geq 0} \mathrm{Q} v \log _{m} x$. Now (20) yields that this group is contained in the smallest convex subgroup $H$ of $v M$ which contains $v x$ and $v \mathcal{R}$. If $w$ is as in our assumption, then $H$ is contained in the convex subgroup $H_{w}$ of $v M$ associated with $w$. Thus, $w$ is trivial on $\mathcal{R}\left(\log _{m} x \mid m \geq 0\right)^{\mathrm{r} \mathrm{R} \mathcal{F}}$, that is, this field lies in $\mathcal{O}_{w}$.

For $\mathcal{F} \subseteq \mathcal{F}_{T}$ we denote by $L E_{\mathcal{R}, \mathcal{F}}(x)$ the smallest subfield of $M$ which contains $\mathcal{R}(x)$ and is real closed and closed under $\mathcal{F}$, exp and log. We shall show how to build up $L E_{\mathcal{R}, \mathcal{F}}(x)$ from $\mathcal{R}(x)$. As a preparation for what we will need in a later paper, we will keep our construction more general. We will construct a variety of fields (described in Lemma 3.8 below) of which $L E_{\mathcal{R}, \mathcal{F}}(x)$ is just a special case. Let $w$ be a convex valuation on $M$, trivial on $\mathcal{R}$, and $H_{w}$ its associated convex subgroup of $v M$. Further, let $K_{0}^{w} \subset \mathcal{O}_{w}$ be any field of the form (18). For example, if $w x=0$, then we can take $K_{0}^{w}=\mathcal{R}\left(\log _{m} x \mid m \geq 0\right)^{\mathrm{rQF}}$. We will see later that if $w \neq v_{\mathcal{R}}$ then there always exists such a field $K_{0}^{w}$ which properly contains $\mathcal{R}$.

Now we construct $K_{1}^{w}$ as follows. Assume that $a \in K_{0}^{w}$ such that $\exp a \notin K_{0}^{w}$, but $v \exp a \in H_{w}$. Then by Lemma 3.5, $K_{0}^{w}(\exp a)^{\mathrm{rQF}}$ is again of the form (18), with $v K_{0}^{w}(\exp a)^{\mathrm{rQ} \mathcal{F}}=v K_{0}^{w} \oplus \mathrm{Q} v \exp a \subset H_{w}$. The latter shows that it is again a subfield of $\mathcal{O}_{w}$. We repeat this procedure until we arrive at a field $K_{1}^{w} \subset \mathcal{O}_{w}$ of the form (18), which contains $\exp a$ for every $a \in K_{0}^{w}$ such that $\exp a \in \mathcal{O}_{w}^{\times}$. Then we construct $K_{2}^{w}$ from $K_{1}^{w}$ in the same way as we constructed $K_{1}^{w}$ from $K_{0}^{w}$. We iterate to obtain fields $K_{n}^{w} \subset \mathcal{O}_{w}$, of the form (18). Their union

$$
K_{\infty}^{w}:=\bigcup_{n \in \mathbb{N}} K_{n}^{w} \subset \mathcal{O}_{w}
$$

is $\mathrm{rQ \mathcal {F }}$-closed and of the form (18). By construction, we have:

Lemma 3.8 $K_{\infty}^{w}$ is the uniquely determined smallest log-and $\mathrm{rQ \mathcal {F }}$-closed subfield of $\mathcal{O}_{w}$, relatively exp-closed in $\mathcal{O}_{w}^{\times}$and containing $K_{0}^{w}$. It is of the form (18).

We derive some further information from our construction. 
Lemma 3.9 Take $n \in \mathbb{N}$. If $a \in K_{n}^{w}$ with $v a<0$, $a>0$, then

$$
v \log a \in v K_{n-1}^{w}, \quad \text { and } \quad v \log _{n} a \in v K_{0}^{w} .
$$

Proof: $\quad$ By the construction of $K_{n}^{w}$ from $K_{n-1}^{w}$, there are elements $a_{j} \in K_{n-1}^{w}, j \in J$, such that $v K_{n}^{w}=v K_{n-1}^{w} \oplus \bigoplus_{j \in J} \mathrm{Q} v \exp a_{j}$. Hence, $a \in K_{n}^{w}$ can be written as

$$
a=\prod_{j \in J_{0}}\left(\exp a_{j}\right)^{q_{j}} \cdot c \cdot r \cdot(1+\varepsilon)
$$

with $J_{0}$ a finite subset of $J, q_{j} \in \mathrm{Q}, c \in K_{n-1}^{w}, r \in \mathbb{R}$ and $\varepsilon \in K_{n}^{w}$ with $v \varepsilon>0$. Then $\log a=\sum_{j \in J_{0}} q_{j} a_{j}+\log c+\log r+\log (1+\varepsilon)$. Since $v \log a<0$ by Lemma 2.4, but $v \log (1+\varepsilon)>0$, we find that $v \log a=v\left(\sum_{j \in J_{0}} q_{j} a_{j}+\log c+\log r\right) \in v K_{n-1}^{w}$. By induction it follows that $v \log _{n} a \in v K_{0}^{w}$.

If $w$ is trivial on $\mathcal{R}$ and $w x=0$ and we start our construction from $K_{0}^{w}=\mathcal{R}\left(\log _{m} x \mid\right.$ $m \geq 0)^{\mathrm{rQF}}$, then $K_{\infty}^{w}$ will be the uniquely determined smallest log- and rQ $\mathcal{F}$-closed subfield of $\mathcal{O}_{w}$, relatively exp-closed in $\mathcal{O}_{w}^{\times}$and containing $\mathcal{R}(x)$. We denote it by

$$
L E_{\mathcal{R}, \mathcal{F}}^{w}(x) .
$$

Let $u$ denote the trivial valuation on $M$. Then $\mathcal{O}_{u}=M$ and $H_{u}=v M$. In this case, $L E_{\mathcal{R}, \mathcal{F}}^{u}(x)$ is exp-closed and contains $x$. Therefore,

$$
L E_{\mathcal{R}, \mathcal{F}}^{u}(x)=L E_{\mathcal{R}, \mathcal{F}}(x) .
$$

Lemma 3.10 Suppose that $x>\mathcal{R}$. Then for every $y \in L E_{\mathcal{R}, \mathcal{F}}(x), y>\mathcal{R}$, the sequence $\exp _{m} y, m \geq 0$, is cofinal in $L E_{\mathcal{R}, \mathcal{F}}(x)$, and the sequence $\log _{m} y, m \geq 0$, is coinitial in $\left\{z \in L E_{\mathcal{R}, \mathcal{F}}(x) \mid z>\mathcal{R}\right\}$.

Proof: It suffices to show the result for $y=x$. Indeed, if it holds in this case, then there is $\nu \in \mathbb{N}$ such that $\exp _{\nu} x>y>\log _{\nu} x$. It follows that $\exp _{n} y>\exp _{\nu+n} x$, $\operatorname{showing}$ that also the sequence $\exp _{m} y, m \geq 0$, is cofinal. It also follows that $\log _{n} x>\log _{\nu+n} y$, showing that also the sequence $\log _{m} y, m \geq 0$, is coinitial.

Take any $a \in L E_{\mathcal{R}, \mathcal{F}}(x), x>\mathcal{R}$. From Lemma 3.9 with $w=u$ and $K_{0}^{w}=\mathcal{R}\left(\log _{m} x \mid\right.$ $m \geq 0)^{\mathrm{rQ \mathcal {F }}}$ we infer that $v \log _{n} a \in v \mathcal{R}\left(\log _{m} x \mid m \geq 0\right)^{\mathrm{rQ} \mathcal{F}}$ for some $n \in \mathbb{N}$. By Lemma 3.7, every element $\alpha<0$ in this value group is either archimedean equivalent to $v x$, or satisfies $v x \ll \alpha<0$. Since $v \log _{n} a \ll v \log _{n+1} a<0$ by Lemma 2.4, it follows that $v x \ll$ $v \log _{n+1} a<0$. Hence by (1), $x>\log _{n+1} a$ and therefore, $\exp _{n+1} x>a$.

Now let $a \in L E_{\mathcal{R}, \mathcal{F}}(x), a>\mathcal{R}$. As before, $v \log _{n} a \in v \mathcal{R}\left(\log _{m} x \mid m \geq 0\right)^{\mathrm{rQF}}$ for some $n \in \mathbb{N}$. As the sequence $v \log _{m} x, m \geq 0$, is cofinal in the negative part of this value group, there is some $m_{0}$ such that $v \log _{n} a<v \log _{m_{0}} x$. Hence by (1), $a \geq \log _{n} a>\log _{m_{0}} x$.

Now we deduce our main theorem on the valuation theoretical structure of $L E_{\mathcal{R}, \mathcal{F}}(x)$. If we take $\mathcal{F}=\mathcal{F}_{T}$ and $M=H(\mathcal{R})$, then $H(\mathcal{R})=L E_{\mathcal{R}, \mathcal{F}}(x)$ by what we have remarked in the introduction, and thus the theorem describes the structure of the Hardy field $H(\mathcal{R})$. 
Theorem $3.11 L E_{\mathcal{R}, \mathcal{F}}(x)$ is of the form

$$
\mathcal{R}\left(x_{i} \mid i \in I\right)^{\mathrm{rQ \mathcal {F }}} \text { with } x_{i}>0 \text { and } v_{\mathcal{R}} x_{i}, i \in I \text {, Q-linearly independent. }
$$

Moreover,

$$
L E_{\mathcal{R}, \mathcal{F}}(x) v_{\mathcal{R}}=\mathcal{R}
$$

The elements $x_{i}$ can be chosen so as to include $x$ and $\log _{m} x$ for all $m \in \mathbb{N}$.

If $\mathcal{R}=\mathbb{R}$, then $L E_{\mathcal{R}, \mathcal{F}}(x)$ has exponential rank 1 , in the sense of [K-K2]. In general, $\operatorname{exprk} L E_{\mathcal{R}, \mathcal{F}}(x)=\operatorname{exprk} \mathcal{R}+1$.

Proof: By our construction, we get that $L E_{\mathcal{R}, \mathcal{F}}(x)$ is of the form (18). Since $\mathcal{F} \subseteq \mathcal{F}_{T}$, we have that $L E_{\mathcal{R}, \mathcal{F}}(x) \subseteq L E_{\mathcal{R}, \mathcal{F}_{T}}(x)$. By definition of the valuation $v_{\mathcal{R}}$, its valuation ring is the convex hull of $\mathcal{R}$ in $M$. As $\mathcal{R}$ is an elementary submodel of $L E_{\mathcal{R}, \mathcal{F}_{T}}(x)$, we can deduce from [D-L], p. 75, (1), that this valuation ring is $T(\exp )$-convex in $L E_{\mathcal{R}, \mathcal{F}_{T}}(x)$. Since the $T$ (exp)-definable closure of $\mathcal{R}(x)$ in $L E_{\mathcal{R}, \mathcal{F}_{T}}(x)$ is equal to $L E_{\mathcal{R}, \mathcal{F}_{T}}(x)$, we can apply Corollary 5.4 of $[\mathrm{D}-\mathrm{L}]$ to obtain that $L E_{\mathcal{R}, \mathcal{F}_{T}}(x) v_{\mathcal{R}}=\mathcal{R}$. Since $\mathcal{R} \subset L E_{\mathcal{R}, \mathcal{F}}(x) \subseteq$ $L E_{\mathcal{R}, \mathcal{F}_{T}}(x)$, this proves $(22)$. By Lemma 3.6, this also implies that $v_{\mathcal{R}} x_{i}, i \in I$, are Q-linearly independent.

The exponential rank is the order type of the set of proper $T(\exp )$-convex valuation rings, ordered by inclusion. Lemma 3.10 shows that $L E_{\mathcal{R}, \mathcal{F}}(x)$ has exactly one more than $\mathcal{R}$, namely $\mathcal{R}$ itself. This proves our assertions about the exponential rank.

\subsection{Levels}

An infinitely increasing unary function $f$ on $\mathcal{R}$ has level $s$ if $s \in \mathbb{Z}$ and there is $N \in \mathbb{N}$ such that $\log _{N+s} \circ f$ is asymptotic to $\log _{N}$ on $\mathcal{R}$. Note that if the latter holds, then it also holds for every integer $N^{\prime}>N$ in the place of $N$. If a denotes the germ of $f$ in $H(\mathcal{R})$, then by (15) the condition is equivalent to

$$
v\left(\frac{\log _{N+s} a}{\log _{N} x}-1\right)>v \mathcal{R} .
$$

Here, $N$ can be chosen such that $N+s \geq 0$. Suppose that $s<s^{\prime} \in \mathbb{Z}$. Since $a>\mathcal{R}$ we have that $v a<v \mathcal{R}$; hence by Corollary 2.5, $v \log _{N+s} a \neq v \log _{N+s^{\prime}} a$ which shows that the above inequality cannot hold for $s^{\prime}$ in the place of $s$. Thus, the level $s$ is uniquely determined (see also $[\mathrm{M}-\mathrm{M}]$ ).

We say that $\mathcal{R}$ is levelled if every $\mathcal{R}$-definable ultimately strictly increasing and unbounded unary function on $\mathcal{R}$ has a level. In this section, we will prove that every definable function on $\mathcal{R}$ has a level, and we will determine this level explicitly.

Take any $a \in L E_{\mathcal{R}, \mathcal{F}}(x)$ such that $a>\mathcal{R}$. According to our construction, we write $L E_{\mathcal{R}, \mathcal{F}}(x)=K_{\infty}$ with $K_{0}=\mathcal{R}\left(\log _{m} x \mid m \geq 0\right)^{\mathrm{rQF}}$. By Lemma 3.9 there is some

$n \in \mathbb{N}$ such that $v \log _{n} a \in v K_{0}$. Similarly as in the proof of Lemma 3.4, we write 
$\log _{n} a=r^{\prime} \prod_{i \geq 0}\left(\log _{i} x\right)^{q_{i}} \cdot r \cdot(1+\varepsilon)$ with $q_{i} \in \mathrm{Q}$, only finitely many of them nonzero, $r^{\prime} \in \mathcal{R}, r \in \mathbb{R}$ and $\varepsilon \in K$ such that $v \varepsilon>0$. It follows that

$$
\log _{n+1} a=\log r^{\prime}+\sum_{i \geq 0} q_{i} \log _{i+1} x+\log r+\log (1+\varepsilon) .
$$

As $a>\mathcal{R}$ by assumption, there must be at least one nonzero $q_{i}$. Let $i_{0}$ be the smallest of all $i \geq 0$ for which $q_{i} \neq 0$. We have that $v \log r=0, v \log (1+\varepsilon)>0$ and $v \log _{i_{0}+1} x<v \log _{i+1} x$ for $i>i_{0}$. Also, $v \log _{i_{0}+1} x<v r^{\prime}$. Thus, we can write $\log _{n+1} a=q_{i_{0}} \log _{i_{0}+1} x \cdot\left(1+\varepsilon^{\prime}\right)$ with $v \varepsilon^{\prime}>0$. Then

$$
\log _{n+2} a=\log q_{i_{0}}+\log _{i_{0}+2} x+\log \left(1+\varepsilon^{\prime}\right) .
$$

Again, $v \log _{i_{0}+2} x<0=v \log q_{i_{0}}<v \varepsilon^{\prime}=v \log \left(1+\varepsilon^{\prime}\right)$. Hence,

$$
v\left(\log _{n+2} a-\log _{i_{0}+2} x\right)=v\left(\log q_{i_{0}}+\log \left(1+\varepsilon^{\prime}\right)\right)=v \log q_{i_{0}}=0 .
$$

Thus,

$$
v\left(\frac{\log _{n+2} a}{\log _{i_{0}+2} x}-1\right)=-v \log _{i_{0}+2} x>v \mathcal{R} .
$$

We have now proved a result which in fact constitutes an abstract notion of levels, without referring to Hardy fields:

Proposition 3.12 Take any element $a \in L E_{\mathcal{R}, \mathcal{F}}(x)$ such that $a>\mathcal{R}$. Then a "has level over $\mathcal{R}$ " in the following sense: there is some $s \in \mathbb{Z}$ and $N \in \mathbb{N}$ such that

$$
v_{\mathcal{R}}\left(\log _{N+s} a-\log _{N} x\right)>v_{\mathcal{R}} \log _{N} x .
$$

Now take any $\mathcal{R}$-definable, ultimately strictly increasing and unbounded function $f$ on $\mathcal{R}$. Let $a$ be the germ of $f$ at infinity. Then $a>\mathcal{R}$. Hence, $a$ is an element of the Hardy field $H(\mathcal{R})=L E_{\mathcal{R}, \mathcal{F}_{T}}(x)$ of $\mathcal{R}$ (where $x>\mathcal{R}$ ). Then $(23)$ shows that $\log _{n+2} f(x)$ and $\log _{i_{0}+2} x$ are asymptotic as functions on $\mathcal{R}$. That is,

$$
\text { the function } f \text { has level } n-i_{0} \text {. }
$$

This proves Theorem 1.1.

\subsection{A maximality property of the $T$-definable closure in the $T(\exp )$-definable closure}

Lemma 3.13 Assume that $T$ has field of exponents $\mathbb{R}$ and that $\mathbb{R} \subset \mathcal{R} \subset M$ are models of $T(\exp )$. Let $x \in M, x>\mathcal{R}$. Then $\mathcal{R}(x)^{\mathcal{F}_{T}}$ (the $T$-definable closure of $\mathcal{R} \cup\{x\}$ in $M$ ) has the following maximality property:

1) $v_{\mathcal{R}} \mathcal{R}(x)^{\mathcal{F}_{T}} \simeq \mathbb{R}$,

2) $\mathcal{R}(x)^{\mathcal{F}_{T}}$ is maximal among all subfields of $L E_{\mathcal{R}, \mathcal{F}_{T}}(x)$ whose value group w.r.t. $v_{\mathcal{R}}$ is archimedean. 
Proof: Assertion 1) follows from Lemma 3.2. In order to prove assertion 2), we show the following: Take any $a \in L E_{\mathcal{R}, \mathcal{F}_{T}}(x) \backslash \mathcal{R}(x)^{\mathcal{F}_{T}}$. Then $v_{\mathcal{R}} \mathcal{R}(x)^{\mathcal{F}_{T}}(a)$ is not archimedean.

By Theorem 3.11 we can write $L E_{\mathcal{R}, \mathcal{F}_{T}}(x)=\mathcal{R}\left(x_{i} \mid i \in I\right)^{\mathcal{F}_{T}}$ with $x_{i}>0$ and $v_{\mathcal{R}} x_{i}$, $i \in I, \mathbb{R}$-linearly independent, and $x$ among the $x_{i}$. As $a \in \mathcal{R}\left(x_{i} \mid i \in I\right)^{\mathcal{F}_{T}}$, there are $x_{i_{1}}, \ldots, x_{i_{n}}(n \geq 1)$ such that $a \in \mathcal{R}\left(x, x_{i_{1}}, \ldots, x_{i_{n}}\right)^{\mathcal{F}_{T}}$, and we choose $n$ minimal with this property. By the Exchange Lemma for o-minimal theories ([P-S]) applied to $T$, we then obtain that

$$
x_{i_{1}} \in \mathcal{R}\left(x, a, x_{i_{2}}, \ldots, x_{i_{n}}\right)^{\mathcal{F}_{T}} .
$$

Suppose that $v_{\mathcal{R}} \mathcal{R}(x, a)^{\mathcal{F}_{T}}=v_{\mathcal{R}} \mathcal{R}(x)^{\mathcal{F}_{T}}$. Then by Lemma 3.2 ,

$$
\begin{aligned}
v_{\mathcal{R}} \mathcal{R}\left(x, a, x_{i_{2}}, \ldots, x_{i_{n}}\right)^{\mathcal{F}_{T}} & =v_{\mathcal{R}} \mathcal{R}(x, a)^{\mathcal{F}_{T}}\left(x_{i_{2}}, \ldots, x_{i_{n}}\right)^{\mathcal{F}_{T}}=v_{\mathcal{R}} \mathcal{R}(x, a)^{\mathcal{F}_{T}} \oplus \bigoplus_{j=2}^{n} \mathbb{R} v_{\mathcal{R}} x_{i_{j}} \\
& =v_{\mathcal{R}} \mathcal{R}(x)^{\mathcal{F}_{T}} \oplus \bigoplus_{j=2}^{n} \mathbb{R} v_{\mathcal{R}} x_{i_{j}}=\mathbb{R} v_{\mathcal{R}} x \oplus \bigoplus_{j=2}^{n} \mathbb{R} v_{\mathcal{R}} x_{i_{j}} .
\end{aligned}
$$

But this does not contain $v_{\mathcal{R}} x_{i_{1}}$. This contradiction to (24) shows that

$$
v_{\mathcal{R}} \mathcal{R}(x, a)^{\mathcal{F}_{T}} \neq v_{\mathcal{R}} \mathcal{R}(x)^{\mathcal{F}_{T}} .
$$

By the Valuation Property ([D-S2], Proposition 9.2) it follows that

$$
v_{\mathcal{R}} \mathcal{R}(x)^{\mathcal{F}_{T}} \underset{\varsubsetneqq}{\varsubsetneqq} v_{\mathcal{R}} \mathcal{R}(x)^{\mathcal{F}_{T}}(a) .
$$

Since $v_{\mathcal{R}} \mathcal{R}(x)^{\mathcal{F}_{T}} \simeq \mathbb{R}$ it follows that $v_{\mathcal{R}} \mathcal{R}(x)^{\mathcal{F}_{T}}(a)$ is not archimedean.

Lemma 3.14 Let $H \subset H(\mathcal{R})$ be a subfield containing $\mathcal{R}(x)$ and closed under compositions and compositional inverses for $v_{\mathcal{R}}$-positive infinite germs (i.e., germs a $\in H$ such that $a>\mathcal{R}$ ). If $H$ is polynomially bounded (i.e., every germ in $H$ is bounded by a power $x^{n}$ for some $\left.n \in \mathbb{N}\right)$, then $v_{\mathcal{R}}(H)$ is archimedean.

Proof: Assume for a contradiction that there is $g \in H(\mathcal{R})$ such that $g>\mathcal{R}$ and $v_{\mathcal{R}} g \ll v_{\mathcal{R}} x$ or $v_{\mathcal{R}} x \ll v_{\mathcal{R}} g$. The former implies that $g>x^{n}$ for all $n \in \mathbb{N}$, a contradiction to the fact that $H$ is polynomially bounded. So assume that $v_{\mathcal{R}} x \ll v_{\mathcal{R}} g$. But this implies that for all $n \in \mathbb{N}$,

$$
x^{n}<g^{-1},
$$

where $g^{-1}$ denotes the compositional inverse of $g$. This again contradicts the assumption that $H$ is polynomially bounded. Indeed, let $n \in \mathbb{N}$. Since $g^{n}<x$, there exists $r \in \mathcal{R}$ (and we may assume $r>1$ ) such that for $a \in \mathcal{R}$ with $a>r$ we have $g(a)^{n}<a$. On the other hand, $g$ is invertible, ultimately. So for $b$ large enough, $g^{-1}(b)=a$ exists with $a>r$. Thus, $g\left(g^{-1}(b)\right)^{n}<g^{-1}(b)$. 
Corollary 3.15 The field $\mathcal{R}(x)^{\mathcal{F}_{T}}$ (i.e., the Hardy field associated with the reduct of $\mathcal{R}$ to the language of $T$ ) is maximal among the polynomially bounded subfields of $H(\mathcal{R})$ which are closed under compositions and compositional inverses for $v_{\mathcal{R}}$-positive infinite germs.

Proof: $\quad$ Let $H$ be a polynomially bounded subfield of $H(\mathcal{R})$ closed under compositions and compositional inverses for $v_{\mathcal{R}}$-positive infinite germs, and containing $\mathcal{R}(x)^{\mathcal{F}_{T}}$. Then by Lemma $3.14, v_{\mathcal{R}} H$ is archimedean. Hence by Lemma 3.13, $H$ cannot be a proper extension of $\mathcal{R}(x)^{\mathcal{F}_{T}}$.

Let us note that there exist polynomially bounded subfields of $H(\mathcal{R})$ which properly contain $\mathcal{R}(x)^{\mathcal{F}_{T}}$. For instance, $\mathcal{R}(x, \log x)^{\mathcal{F}_{T}}$ and $\mathcal{R}\left(\log _{m} x \mid m \geq 0\right)^{\mathcal{F}_{T}}$ are such fields. But they are not closed under compositions and compositional inverses for $v_{\mathcal{R}}$-positive infinite germs.

\subsection{A maximality property of the Hardy field $H\left(\mathcal{R}_{\text {an,powers }}\right)$}

Now we consider the special case where $\mathcal{F}_{T}$ is the set of function symbols for 0 -definable functions in $\mathbb{R}_{\text {an,powers }}$. We let $\mathcal{R}_{\text {an,powers }}$ denote the reduct of $\mathcal{R}$ to the language of $\mathbb{R}_{\text {an,powers }}$, and $\mathcal{R}_{\text {an,exp }}$ the reduct of $\mathcal{R}$ to the language of $\mathbb{R}_{\text {an,exp }}$. Since

$$
x^{r}=\exp (r \log x)
$$

for all $r \in \mathbb{R}$, the power functions are $\mathbb{R}$-definable (actually, already 0-definable) in $\mathcal{R}_{\text {an,exp }}$. Therefore,

$$
H\left(\mathcal{R}_{\text {an,exp }}\right)=H(\mathcal{R})
$$

On the other hand, $H\left(\mathcal{R}_{\text {an,powers }}\right)$ is a proper subfield of $H(\mathcal{R})$. It has the following maximality property:

Theorem 3.16 Let $H \subseteq H(\mathcal{R})$ be a polynomially bounded field containing $H\left(\mathcal{R}_{\text {an,powers }}\right)$ and closed under compositions and compositional inverses for $v_{\mathcal{R}}$-positive infinite germs. Then $H=H\left(\mathcal{R}_{\text {an,powers }}\right)$.

In particular, $H\left(\mathcal{R}_{\text {an,powers }}\right)$ is maximal among the Hardy subfields of $H(\mathcal{R})$ associated with polynomially bounded reducts of $\mathcal{R}$.

Proof: We take $T$ to be the elementary theory of $\mathcal{R}_{\text {an,powers. }}$ We know that $H\left(\mathcal{R}_{\text {an,powers }}\right)=$ $\mathcal{R}(x)^{\mathcal{F}_{T}}$ with $x \in H(\mathcal{R}), x>\mathcal{R}$ the germ of the identity function. Now our first assertion follows from Corollary 3.15.

If $H$ is the Hardy field of a polynomially bounded reducts of $\mathcal{R}$, then $H$ is closed under compositions and compositional inverses for $v_{\mathcal{R}}$-positive infinite germs. Hence our second assertion follows from the first.

\section{References}


[C-K] Chang, C. C. - Keisler, H. J. : Model Theory, Amsterdam - London (1973)

[D] van den Dries, L.: T-convexity and Tame Extensions II, J. Symb. Logic 62 (1997), 14-34

[D-L] van den Dries, L. - Lewenberg, A. H.: T-convexity and tame extensions, J. Symb. Logic 60 (1995), 74-102

[D-M-M1] van den Dries, L. - Macintyre, A. - Marker, D.: The elementary theory of restricted analytic functions with exponentiation, Annals of Math. 140 (1994), $183-205$

[D-M-M2] van den Dries, L. - Macintyre, A. - Marker, D. : Logarithmic-Exponential Power Series, J. London Math. Soc. 56 (1997), 417-434

[D-S1] van den Dries, L. - Speissegger, P.: The real field with convergent generalized power series, Trans. Amer. Math. Soc. 350 (1998), 4377-4421

[D-S2] van den Dries, L. - Speissegger, P.: The field of reals with multisummable series and the exponential function, Proc. London Math. Soc. 81 (2000), 513565

[KA] Kaplansky, I. : Maximal fields with valuations I, Duke Math. Journ. 9 (1942), 303-321

[K-K1] Kuhlmann, F.-V. - Kuhlmann, S.: On the structure of nonarchimedean exponential fields II, Comm. in Algebra 22(12) (1994), 5079-5103

[K-K2] Kuhlmann, F.-V. - Kuhlmann, S.: The exponential rank of nonarchimedean exponential fields, in: Proceedings of the Special Semester in Real Algebraic Geometry and Ordered Structures, Baton Rouge 1996, Contemporary Mathematics 253 (2000), 181-201

[K-K3] Kuhlmann, F.-V. - Kuhlmann, S.: Residue fields of arbitrary convex valuations on restricted analytic fields with exponentiation I, The Fields Institute Preprint Series (1996)

[K-K-S] Kuhlmann, F.-V. - Kuhlmann, S. - Shelah, S. : Exponentiation in power series fields, Proc. Amer. Math. Soc. 125 (1997), 3177-3183

[KS1] Kuhlmann, S.: On the structure of nonarchimedean exponential fields I, Archive for Math. Logic 34 (1995), 145-182

[KS2] Kuhlmann, S.: Ordered Exponential Fields, The Fields Institute Monographs 12, AMS Publications (January 2000)

[M-M] Marker, D. - Miller, C.: Levelled o-minimal structures, in: Real algebraic and analytic geometry (Segovia, 1995), Rev. Mat. Univ. Complut. Madrid 10 (1997), 241-249

[M] Miller, C. : Expansions of the real field with power functions, Ann. Pure Appl. Logic 68 (1994), 79-94

[P-S] Pillay, A. - Steinhorn, C.: Definable sets in ordered structures I, Trans. Amer. Math. Soc. 295 (1986), 565-592

[R] Ribenboim, P.: Théorie des valuations, Les Presses de l'Université de Montréal, Montréal, 1st ed. (1964), 2nd ed. (1968)

Mathematical Sciences Group, University of Saskatchewan,

106 Wiggins Road, Saskatoon, Saskatchewan, Canada S7N 5E6

email: fvk@math.usask.ca — home page: http://math.usask.ca/ fvk/index.html

email: skuhlman@math.usask.ca — home page: http://math.usask.ca/ skuhlman/index.html 